\title{
Hypertension, hypotension, longevity and dementia
}

\author{
Enrico Mossello \\ Department of Experimental and Clinical Medicine, University of Florence, and Division of Geriatric Medicine and \\ Intensive Care Unit, Azienda Ospedaliero-Universitaria Careggi, Florence, Italy
}

\begin{abstract}
High blood pressure at midlife has been consistently identified as a risk factor for dementia in late life, while dementia onset is typically associated with a subsequent decline of blood pressure values. A previous meta-analysis of randomized controlled studies of antihypertensive treatment in old age has shown a borderline effect of active treatment in reducing the risk of dementia. The cognitive substudies of SPRINT (SPRINT-MIND) and of HOPE-3, published in 2019, were aimed at assessing the cognitive effect of aggressive antihypertensive treatment. In SPRINT-MIND, that included subjects with high vascular risk, the risk of dementia (primary outcome) did not differ between groups, but the risk of mild cognitive impairment was significantly reduced in the treatment group. Conversely in HOPE-3, that included subjects with intermediate vascular risk, no significant cognitive effect was observed, with a trend for a better outcome in the placebo arm in the subgroup with lower baseline systolic blood pressure. These data add to observational studies showing detrimental cognitive effect of lower blood pressure values in very old subjects, with cognitive impairment, disability, and complex health problems. Regarding longevity, observational studies confirm protective effects of lower blood pressure values, although systolic blood pressure $<130 \mathrm{mmHg}$ are associated with greater mortality risk in subjects with cognitive and/or motor impairment. On the whole antihypertensive treatment might decrease the risk of cognitive impairment in older, robust, high vas-
\end{abstract}

Correspondence: Enrico Mossello, Department of Experimental and Clinical Medicine, University of Florence, Viale G. Pieraccini 6, 50139 Firenze, Italy.

Tel. +39.055.7949429.

E-mail: enrico.mossello@unifi.it

Keywords: Hypertension; dementia; neurocognitive disorders; hypotension; antihypertensive agents.

Conflict of interest: The author declares no conflict of interest.

Received for publication: 21 February 2020.

Accepted for publication: 11 November 2020.

${ }^{\circ}$ Copyright: the Author(s), 2020

Licensee PAGEPress, Italy

Monaldi Archives for Chest Disease 2020; 90:1674

doi: $10.4081 /$ monaldi.2020.1674

This article is distributed under the terms of the Creative Commons Attribution Noncommercial License (by-nc 4.0) which permits any noncommercial use, distribution, and reproduction in any medium, provided the original author(s) and source are credited. cular risk subjects. Yet the presence of cognitive impairment might modify the prognostic effect of antihypertensive treatment and advise against aggressive blood pressure lowering.

\section{Blood pressure and dementia risk in life course}

During last 20 years, several longitudinal studies have identified an association between blood pressure values at midlife and cognitive decline and/or dementia [1]. More recently, in the US Atherosclerosis Risk in Communities cohort, a dose-response relationship was observed between systolic blood pressure (SBP) at midlife, especially in untreated hypertensives, and cognitive deterioration over a 20-year follow-up. Of notice, no association was observed between BP at the end of follow-up and cognitive decline [2]. Lifetime studies, which have followed blood pressure and cognitive function trajectories for over 30 years, have confirmed this bimodal association, as subjects bound to develop dementia in old age had higher blood pressure values until the age of 75, especially if not receiving antihypertensive drugs, and then showed a sharper blood pressure decline than those maintaining a normal cognitive function [3]. These data suggest that the potential "window of opportunity" for antihypertensive treatment preventing subsequent cognitive decline possibly ranges from midlife to "young old" age and that long-term antihypertensive treatment is possibly needed to observe a beneficial effect.

\section{Randomized controlled trial of antihypertensive treatment with cognitive outcome}

The long-time course needed to observe a clear association between high blood pressure and dementia risk hinders the observation of a consistent effect of cognitive protection in randomized controlled trials of antihypertensive treatment in old age, that have been typically powered to detect a reduction of major vascular events and mortality over shorter time periods.

The open label extension of the randomized controlled Syst-Eur was the only single study showing a statistically significant benefit of antihypertensive treatment against dementia, with a $55 \%$ decreased incidence at 4-year follow-up [4]. The placebo-controlled HYVET study included subjects aged $80+$ with isolated systolic hypertension and was stopped after only 2 years only for the clear superiority of active treatment on vascular outcomes and all-cause mortality, thus being unable to show a significant effect on cognition [5]. The meta-analysis of Syst-Eur, HYVET and other available placebo-controlled trials showed a borderline significant, protective effect of antihypertensive treatment against incident dementia, with large heterogeneity among different studies [5]. 
In 2019 the cognitive-sub-studies of two large randomized controlled trials have been published. SPRINT trial had previously shown the efficacy of an aggressive antihypertensive treatment (target $<120 \mathrm{mmHg}$ ) over a standard treatment $(<140 \mathrm{mmHg})$ in reducing the risk of a composite endpoint of cardiovascular mortality plus major cardiovascular events in a population with high vascular risk, but excluding subjects with previous stroke, diabetes mellitus, heart failure, orthostatic hypotension, and dementia [6]. For this reason the trial was stopped prematurely after 3.2 years, but open-label follow-up went-on, including the secondary preplanned cognitive outcome, that was the objects of the SPRINTMIND sub-study [7]. Among 8563 subjects included in this study (mean age 67, mean SBP $140 \mathrm{mmHg}$, mean BMI 30) no significant between-group difference was observed in the primary outcome of dementia onset after a 5.1-year follow-up. Yet a significant difference was observed between treatment and placebo group for the onset of mild cognitive impairment and the composite outcome of mild cognitive impairment plus dementia, with a $15 \%$ risk reduction estimate [7]. Conversely, in HOPE-3 trial, the addition of an antihypertensive treatment (candesartan plus hydrochlorothiazide) to standard treatment of participants at intermediate risk, who did not have cardiovascular disease and had not a clear indication to renin-angiotensin axis inhibition, did not show any effect on the primary outcome of cardiovascular mortality plus major cardiovascular events [8]. A group of 1,626 subjects aged 70+ (mean age 74, mean SBP 140, mean BMI 27) was included in the cognitive substudy, that showed no effect on the pre-specified cognitive outcome (performance in the Digit-Symbol Substitution Test) after a 5.7-year follow-up [9]. Moreover, in a subgroup analysis, a lower cognitive decline was observed in the placebo arm among subjects with lower baseline SBP $(<133 \mathrm{mmHg})$, with a significant blood pressure/treatment group interaction [9]. This might suggest that in an older population with a lower risk profile compared with SPRINT, aggressive blood pressure lowering might not be beneficial for cognition, or even potentially harmful.

\section{Blood pressure and cognitive outcome: effect modifiers in cohort studies}

Due to uncertainties deriving from randomized controlled trials, finding of observational studies are of interest, especially those focusing on the effect modifiers of the association between blood pressure and cognitive outcome. The association of blood pressure with cognitive decline and Alzheimer's disease at older ages is uncertain and several data, including a meta-analysis [10], suggest that it might be even reverted in comparison with younger age. Comparing the data from two Dutch epidemiological studies, an association between high blood pressure and cognitive decline after an 11-year follow-up has been observed for subjects aged 6575 , while the reverse association has been observed for subjects aged 85 after 5 years [11].

Biological aging and functional status might modulate the BPcognition association in old age. Indeed, in subjects aged $85+$ years, the protective effect of higher BP on cognition was particularly evident among subjects who already showed a baseline disability in activities of daily living [12]. In an Italian clinical sample of 172 subjects with dementia or mild cognitive impairment aged $65+$ (mean age 79, mean Mini Mental State Examination 22/30), in whom BP was assessed with 24-hour ambulatory monitoring, the risk of cognitive decline at 9 months was increased in subjects with lower mean daytime SBP $(<129 \mathrm{mmHg})$ actively treated with anti- hypertensive drugs [13]. Consistent with these data, in a recently published study on 1,266 subjects aged $75+$ in primary care (mean age 82, mean Mini Mental State Examination 27/30) the association between baseline SBP and cognitive decline after 1 year differed according to health status and ongoing antihypertensive treatment [14]. In fact $\mathrm{SBP}<130 \mathrm{mmHg}$ was associated with greater cognitive decline at follow-up only among subjects identified as having "complex health problems" (functional, somatic, mental, social) and treated with antihypertensive drugs [14].

\section{Cognitive impairment as modifier of prognostic effect of antihypertensive treatment}

A further issue regarding the role of cognition in antihypertensive treatment choice is associated with the assessment of patient's vulnerability. In fact, it has been proposed that the presence of motor and cognitive impairment might be markers of increased susceptibility to adverse events associated with antihypertensive treatment [15]. In particular, the large group of older subjects already suffering from cognitive impairment might represent a subpopulation at high risk for brain hypoperfusion. In fact in a Swedish population-based study no association was observed between blood pressure and mortality in 3,014 older subjects (mean age 73, 13\% with cognitive impairment defined as Mini Mental State Examination $\leq 26$ and/or dementia diagnosis). Yet stratifying for motor impairment (gait velocity $<0.8 \mathrm{~m} / \mathrm{sec}$ ) and/or cognitive decline, impaired subjects showed the greatest mortality in the subgroup with $\mathrm{SBP}<130 \mathrm{mmHg}$, while unimpaired subjects showed the lowest mortality in the SBP $<130 \mathrm{mmHg}$ subgroup [16]. On the other hand, in a large Medicare cohort of older subjects treated with antihypertensive drugs, blood pressure values were not associated per se with an increased risk of fall, while the presence of cognitive impairment was. On the whole, available data caution against an excessive blood pressure lowering among subjects with cognitive impairment, due to uncertainties about beneficial effects and increased risk of adverse events [17].

\section{Conclusions}

Available data support the long-term risk of cognitive impairment associated with high blood pressure. Antihypertensive treatment might reduce the risk of cognitive decline also when started in old age, at least in subjects with a high vascular risk profile and without "frailty conditions", including ongoing functional or cognitive impairment. Conversely, when cognitive impairment is already present, it may negatively interact with low blood pressure values and, most important, with antihypertensive treatment, with several data suggesting that reducing SBP under $130 \mathrm{mmHg}$ might not be advisable in this population. Optimal blood pressure target in cognitively impaired older subjects still needs to be identified.

\section{References}

1. Qiu C, Winblad B, Fratiglioni L. The age-dependent relation of blood pressure to cognitive function and dementia. Lancet Neurol 2005;4:487-99. 
2. Gottesman RF, Schneider AL, Albert M, et al. Midlife hypertension and 20-year cognitive change: the atherosclerosis risk in communities neurocognitive study. JAMA Neurol 2014;71: 1218-27.

3. Joas E, Backman K, Gustafson D, et al. Blood pressure trajectories from midlife to late life in relation to dementia in women followed for 37 years. Hypertension 2012;59:796-801.

4. Forette F, Seux ML, Staessen JA, et al. The prevention of dementia with antihypertensive treatment: new evidence from the Systolic Hypertension in Europe (Syst-Eur) study. Arch Intern Med 2002;162:2046-52.

5. Peters R, Beckett N, Forette F, et al. Incident dementia and blood pressure lowering in the Hypertension in the Very Elderly Trial cognitive function assessment (HYVET-COG): a double-blind, placebo controlled trial. Lancet Neurol 2008;7:683-9.

6. Wright JT, Williamson JD, Whelton PK, et al. A randomized trial of intensive versus standard blood-pressure control. N Engl J Med 2015;373:2103-16.

7. Williamson JD, Pajewski NM, Auchus AP, et al. Effect of intensive vs standard blood pressure control on probable dementia: A randomized clinical trial. JAMA 2019;321:553-61.

8. Lonn EM, Bosch J, López-Jaramillo P, et al. Blood-pressure lowering in intermediate-risk persons without cardiovascular disease. N Engl J Med 2016;374:2009-20.

9. Bosch J, O'Donnell M, Swaminathan B, et al. Effects of blood pressure and lipid lowering on cognition: Results from the HOPE-3 study. Neurology 2019;92:e1435-46.
10. Power MC, Weuve J, Gagne JJ, et al. The association between blood pressure and incident Alzheimer disease: a systematic review and meta-analysis. Epidemiology 2011;22:646-59.

11. Euser SM, van Bemmel T, Schram MT, et al. The effect of age on the association between blood pressure and cognitive function later in life. J Am Geriatr Soc 2009;57:1232-7.

12. Sabayan B, Oleksik AM, Maier AB, et al. High blood pressure and resilience to physical and cognitive decline in the oldest old: the Leiden 85-plus Study. J Am Geriatr Soc 2012;60:2014-9.

13. Mossello E, Pieraccioli M, Nesti N, et al. Effects of low blood pressure in cognitively impaired elderly patients treated with antihypertensive drugs. JAMA Intern Med 2015;175:578-85.

14. Streit S, Poortvliet RKE, Elzen WPJD, et al. Systolic blood pressure and cognitive decline in older adults with hypertension. Ann Fam Med 2019;17:100-7.

15. Mossello E, Desideri G, Ungar A. Hypertension in the oldest old, beyond guidelines.In: A. Ungar, N. Marchionni, editors. Cardiac management in the frail elderly patient and the oldest old. Cham: Springer; 2017. pp- 87-103.

16. Liang Y, Fratiglioni L, Wang R, et al. Effects of biological age on the associations of blood pressure with cardiovascular and non-cardiovascular mortality in old age: A population-based study. Int J Cardiol 2016;220:508-13.

17. Bromfield SG, Ngameni CA, Colantonio LD, et al. Blood pressure, antihypertensive polypharmacy, frailty, and risk for serious fall injuries among older treated adults with hypertension. Hypertension 2017;70:259-66. 